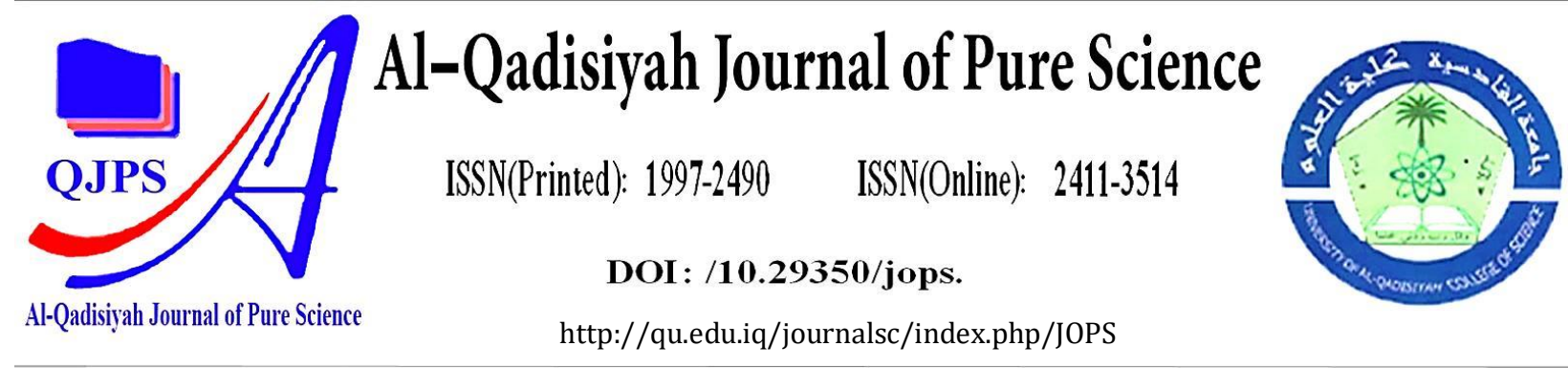

\title{
Inhibition growth of Aspergillus niger that isolated from some fodder grains by using the Pleurotus ostreatus and Calcium citrate
}

\section{Authors Names \\ a. Mohammed H. Halim \\ b. Abdul_ameer S. Saadon \\ Article History}

Received on: $12 / 7 / 2020$

Revised on: 29/7/2020

Accepted on: 6/8/2020

\section{Keywords:}

Aspergillus niger , PCR, Pleurotus ostreatus .

DOI: https://doi.org/10.293 50/jops.2020.25.3.1164

\begin{abstract}
Samples were collected from the stores, fields and local markets of Diwaniya province for the purpose of study from August / October 2019, the following fungi were isolated: Aspergillus niger, Aspergillus flavus, Aspergillus ochraceus, Aspergillus fumigatus, Penicillium natatum, Rhizopus stolanifer, Alternaria Alternata, Fusarium solani, Fusarium proliferatum, Fusarium sp. Tricoderma sp. . Some significant differences were observed in the frequency of fungi isolated from fodder grains, the frequency percentage highest in the treatment of nonsterilized grains reach $15.23 \%$ for the A. niger fungus and in sterilized grain about $13.88 \%$. A.niger diagnosed phenotypic using classification keys and molecular diagnosed with PCR technique (polymerase chain reaction) with sequencing analysis and phylogenic tree analysis for the purpose of comparing A.niger fungi isolates with some global strains . The effect of p.ostreatus showed ability of this fungus to inhibit growth of A.niger fungus when the antagonism occur between two fungus. Interference between p.ostreatus filtrate concentration and calcium citrate effect in the radial growth of the this fungus showed a clear decrease in the level of colonial diameters, where the inhibition percent reached to $71.79 \pm 0.06 \%$ at concentration $30 \%$.
\end{abstract}

\section{Introduction}

The contamination of some fodder, grains and in general foods with fungi and their toxins are problems that threaten most developed countries, especially those that lack perfect storage conditions and are a source of concern, which called on these countries to provide safe, healthy food sources free from contamination to achieve food security and thus not to harm the animal, humans are from these toxins [1] . It is shown that the survey study of 28 storge fungi, most of them belonging to two species, Aspergillus and Penicillium accompanied with the stored grains, were isolated from samples representing 24 types of wheat, barley, yellow corn, and rice stored in northern, central and southern Iraq [2] . The fungus Aspergillus and Fusarium attack grains during storage, these fungi may continue with the grains until they reach to the animal and cause great harm [3]. Some species of the genus Aspergillus are opportunistic fungi such as A.parasiticus, A flavus, it causes diseases for people with weak immune systems, called Aspergillosis, as the fungus grows in the form of cavities caves inside the lung, and its symptoms are similar to tuberculosis, it may also cause otitis [4] . The colonies grow clearly and quickly on the PDA medium, the colonies appear soft or slightly mystical 
with a black color resulting from the formation of black spores in large numbers and the colony diameter is between (5-6) $\mathrm{cm}$ after a short period of incubation [5]. Inhalation of large quantities of A.niger causes numerous dangerous and highly sensitive human lung diseases such as asthma and pneumonia [6] . A. niger also produces many Mycotoxins that have an effect on human and animal health such as aflatoxin, oxatoxin, citrinin and other toxins [7]. Therefore, there are several treatment agents that have been tested to control A.niger fungus. In this study, select A.niger because prevalence this fungus in poultry and birds feeds and two factors were suggested the first biological is Pleurotus ostreatus filtrate and the second is the citrate calcium as a chemically agent to control it .

\section{Material and methods}

\section{Sample collection :}

Sample of poultry and bird feeds were collected from Diwaniya province stores and local markets by an appropriate amount according to the type of sample for laboratory testing for each sample and placed in special bags for preservation and transferred to the laboratory, the exposed ones were washed well with sterile water and left to dry at room temperature and kept in the refrigerator at $4^{\circ} \mathrm{C}$ until use , samples taken included poultry feed, pellet feed, green poultry feed, yellow corn, white corn, as well as local millet in the city markets for the period from (August - October 2019) .

\section{Cultures Media Preparation :}

\section{Potato's Dextrose Agar (PDA) :}

Prepare this medium according to the manufacturer's specifications by dissolving $39 \mathrm{~g}$ of it in $1000 \mathrm{ml}$ of distilled water, then sterilize the medium with autoclave at $121^{\circ} \mathrm{C}$ and under 15 pounds pressure for 15 minutes. Before cooling the medium, add the antibiotic Chloramphenicol at $250 \mathrm{mg} / 1$ for the purpose of Preventing bacterial growth. Use this medium to isolate and keeping fungi [8] .

\section{Isolate the fungi associated with contaminated fodder grains :}

Contaminated fungi were isolated from fodder grains that were used in the research . Each sample was divided into two groups. The first one are included samples that were sterilized by using sodium hypochlorite superficially with a concentration of $1 \%$ for a period of 3 minutes and then washed with distilled water three times to remove the sterilization effect [9], the second was washed with distilled water only, then took parts of sterile and non-sterile samples by sterile forceps, culture in a Petri dish containing the PDA medium, three replicates for each sample, then incubated for 5 days at a temperature of $25^{\circ} \mathrm{C}$. During this period the growth of fungi [10] was followed up and observed developing fungi, after being diagnosed by calculating the percentage of frequency .

\section{Diagnosis of isolated fungi :}

Microscopic and Phenotypic examination :

Diagnosis of fungi isolated from the feed grains after the isolation and purification, the diagnosis to the genus and species levels depending on the appearance of the colony morphological features such as color, shape, its base, also depend on microscopic features such as the size of conidiophore , spores, their shape and arranged according to the taxonomic bases approved using the classification keys provided in $[11,12]$.

\section{Primers :}

In this study, one type of gene Primer ( ITS region 18S rRNA ) was used for diagnosing the fungus Aspergillus niger it was designed from the Genbank NCBI website using Primer 3 Plus .

\section{Table ( 1 ) : Primers DNA Reverse \& Forward that used for A.niger diagnosis .}

\begin{tabular}{|c|c|c|c|}
\hline N. & Secquence Nucleotide & Product size pb & Origin \\
\hline F. & 5'-GGAAGGGRTGTATTTATTAG-3 & 1500 & $\begin{array}{c}\text { Designed for this } \\
\text { experiment }\end{array}$ \\
\cline { 1 - 2 } R. & 5- TCCTCTAAATGACAAGTTTG-3 & 1500 \\
\hline
\end{tabular}


Molecular diagnosis of $A$. niger by polymerase chain reaction technique :

The PCR method consists of several steps :

A- DNA Extraction from A.niger : to complete the extract of DNA from A.niger, use a special kit, which is the (Prep Fungi / Yeast Genomic DNA Extraction Mini Kit) and by following these steps :

1- Activation the strains of the fungus, taking part of the edge of the fungal culture of the growing fungus and five days old on the medium of the PDA .

2- Take $1 \times 10^{6}$ spore / $\mathrm{ml}$ of fungal culture to a micro centrifuge $1.5 \mathrm{ml}$ tube, with the addition of $1 \mathrm{ml}$ of FA Buffer to the fungal cells stuck by Micropipette and the fungal cells were deposited by Centrifuge at $5000 \mathrm{r} / \mathrm{min}$ for two minutes .

3- Taken from the fungal cells precipitate, FB550 $\mu 1$ solution was added and $50 \mu 1$ from the lyticase enzyme was added, well mixed by Vortexing apparatus then samples were incubated at $37{ }^{\circ} \mathrm{C}$ for 30 minutes, and $8 \mathrm{ml}$ at a concentration of $50 \mathrm{mg} / \mathrm{ml}$ RNase was added. And incubate for two minutes at room temperature .

4- Place the cell mixture in the centrifuge at $5000 \mathrm{r} / \mathrm{min}$ for ten minutes, then the supernatant was removed .

5- Added TG1 buffer $350 \mu \mathrm{l}$ and mix well, using micro pipetting, transfer the sample mixture to a second new collection tube, and mix well using vortexing for $5 \mathrm{~min}$. .

6- Added $20 \mu \mathrm{l}$ Proteinase $\mathrm{K}(10 \mathrm{mg} / \mathrm{ml})$ and mix well by vortexing, incubate at $55^{\circ} \mathrm{m}$ for 15 minutes.

7- The cells were deposited by the centrifuge at $5000 \mathrm{r} / \mathrm{min}$ for one minute and transfer $(200 \mu \mathrm{l})$ from the floating mixture to a microcentrifuge $1.5 \mathrm{ml}$ new tube and $200 \mu \mathrm{l}$ TG2 Buffer was added and mixed well using Pipetting .

8- $200 \mu \mathrm{l}$ (96-100\%) ethanol was added and mixed well for 10 seconds, put the TG Mini Column into the Collection Tube, and transfer the sample mixture carefully to the TG Mini . $400 \mu 1$ of W1 Buffer was added to the TG Mini Column and Centrifuge at $11000 \mathrm{x}$ g for 30 seconds.

9- Added $750 \mu \mathrm{l}$ of Wash Buffer to the TG Mini Column and centrifuge at $11000 \mathrm{x}$ g for 30 seconds, placing the TG Mini Column in the Elution Tube.

10- $50 \mu$ l of Elution Buffer was added to the membrane center of TG Mini Column, stopping TG Mini Column for 3 minutes and centrifuged at 18,000 $\mathrm{x}$ g for one minute for total DNA .

11- Preservation of total DNA at $-20^{\circ} \mathrm{C}$.

\section{B- Measured purification and concentration DNA :}

The DNA extracted from A.niger strains was detected by using a Nano drop spectrophotometer (THERMO. USA) device to detect and measure the concentration of DNA and RNA by identifying the DNA concentration $(\mathrm{ng} / \mu \mathrm{l})$ and measuring the DNA purity through reading absorbance with a wavelength of between (260 -280) nanometers .

\section{C- Double Amplification DNA :}

The reaction (AMB PCR Master Mix) was prepared for the PCR, where $1 \mu 5$ of the extracted DNA and $1 \mu 4$ of each forward and $2 \mu 1$ reverse $(1 \mu 2)$ were added to each AMB PCR. Complete the volume to $1 \mu 25$ by adding PCR water. The new components using the Vortex mixer were then placed in the Thermocycler device for the PCR reaction to conduct the DNA amplification process according to the optimal conditions of the thermal cycles mentioned in the following table (2), [13] .

Table ( 2 ) : Optimum conditions for thermal cycles of PCR reaction .

\begin{tabular}{|c|c|c|c|}
\hline PCR Step & Repeat cycle & Temperature C $^{\circ}$ & Time \\
\hline Initial denaturation & 1 & 95 & $5 \mathrm{~min}$ \\
\hline Denaturation & & 95 & $30 \mathrm{sec}$ \\
\hline Annealing & \multirow{2}{*}{30} & 58 & $30 \mathrm{sec}$ \\
\hline Extension & 1 & 72 & $45 \mathrm{sec}$ \\
\hline Final extension & & 72 & $7 \mathrm{~min}$ \\
\hline
\end{tabular}


Forever

\section{D- Gel electrophoresis :}

The Agarose Gel was prepared using the method [14, added $3 \mu 1$ of dye Ethidium bromide used in DNA staining, poured in the place designated for it in the electrophoresis and put the sterile comb into the mixture to make holes in the gel and leave for 40-30 minutes at room temperature for the gel to solidify, then the comb was removed and $5 \mu 1$ of Ladder was added to the hole The first containing pieces of standard DNA, the same quantities of extracted nucleic acids were added to the others pits at 70v for an hour and for the purpose of detecting the amplified extracted DNA bundles that represent PCR and compared to the standard Ladder and after completing the electrophoresis of the samples the gel layer containing the DNA was examined under UV rays and images were taken .

\section{E - DNA sequencing analysis :}

The DNA sequence of the ITS1-18S rRNA gene region of A.niger fungus was obtained by sending the product PCR reaction to the University of Seoul / in South Korea for the purpose of examining the nucleotide sequence, areas of genetic variation and genetic distances were extracted and phylogenetic tree analysis performed using the MEGA 10 program to comparing the DNA sequence of local A.niger strains with the DNA sequence of some global A.niger fungi recorded in the NCBI database .

\section{Biological and chemical agents treatment :}

The filtrate of P.ostreatus was prepared by using the PDB in flasks with an amount of $100 \mathrm{ml}$ from the medium and sterilized by autoclave and before cooling ,Chloramphenicol was added to the medium and then taking two pieces diameter of $5(\mathrm{~mm})$ From fungus grown on PDA at the age of 7 days in each flask. Flasks incubated in the incubator at a temperature of $25^{\circ} \mathrm{C}$. for a period of three weeks with continuous shaking every two days. After period ended, the fungal cultures were filtered for the fungus using Whatman No. 1 . Under sterile conditions and then sterilize the filtrate using Millipore filters with a diameter of 0.22 microns . Calcium citrate salt was prepared according to instructions of the producing company .

\section{Effect of $P$. ostreatus on the growth of A.niger contaminated fungus on PDA (Antagonism) :}

Double culture technique was used for the two fungus in $9 \mathrm{~cm}$ diameter petri dishes containing the PDA solid medium . To find and to reveal the oppositional ability of the P. ostreatus and the contaminated A. niger, the plate was divided into two equal halves. By a cork Borer take disk of 5 millimeter of $P$. ostreatus at the age of seven days, and made a hole in the center of the second half of the petri and with a disk of $5 \mathrm{~mm}$ of a A.niger fungus at the age of 7 days and at three replicates. At a temperature of $25^{\circ} \mathrm{C}$. for a period of 7 days, after the incubation was over, calculated the degree of antagonism for each fungus, according to the five-step standardization scale mentioned by [15] , which consisted of five degrees : (1) Resistant fungus covering the entire plate . (2) Resistant fungus $3 / 4$ cover the area of the plate . (3) Both fungus cover half the surface of the plate . (4) Pathogenic fungus cover $3 / 4$ of the plate. (5) The pathogenic fungus covers the entire plate .

\section{Effect of P.ostreatus concentrations on the radial growth of A.niger Pathogenic :}

To determine effectiveness of the P.ostreatus in the radial growth of A. niger followed [16] , a Poisoned Food Technique, three different concentrations of P.ostreatus filtrate were prepared, which is $10,20,30 \%$ add to PDA sterilized prepared, shake the medium circularity motion then left the plates to solidify and by the cork borer a hole was made in the middle of the petri , transferred a piece measuring (5) $\mathrm{mm}$ from the end of the radial growth of the A.niger fungus at the age of 7 days to the center of the petri and with three replications for each concentration and placed in the incubator for a period of seven days at a temperature of $25^{\circ} \mathrm{C}$. The control plates were left without any addition . After the end of the incubation peroid, the growth rate of the fungus was measured in the treatments for the different concentrations $10,20,30 \%$. The measurement of the growth rate of the fungus in the plates was taken using a ruler after the Mycelium reaches the edge of the petri in the control treatment, 
radial growth was calculate by taking the growth rate for two the developing colonies then calculate percent inhibition of radial growth applying the formula mentioned by [17] and used by [18] .

\section{The effect of calcium citrate concentrations on the radial growth of $A$. niger fungus :}

To determine the effectiveness of the calcium citrate in the radial growth of $A$. niger, Dixit et al (1976) followed the Poisoned Food Technique method, as this salt was prepared according to the manufacturer's instructions, and with three concentrations of calcium citrate salt 10,20,30\%, and these different concentrations of this salt are added with the sterile culture medium (PDA), and the same steps mentioned in the effect of the P.ostreatus concentrations were followed above, these two factors were used together to reduce or inhibit growth of A.niger .

The effect of interfere calcium citrate and P.ostreatus filtrate on the radial growth of $A$. niger : To determine the effect of calcium citrate and P.ostreatus filtrate on the radial growth of $A$. niger fungus, the interference process was performed and as shown :

1) $10 \%$ of P. ostreaus filtrate $+10 \%$ calcium citrate. 2) $20 \%$ of $P$. ostreaus filtrate $+20 \%$ calcium citrate. 3) $30 \%$ of P. ostreaus filtrate $+30 \%$ calcium citrate. As for the control treatment, PDA was used without any addition with the same steps as in previous treatments .

\section{Results and discussion}

\section{Isolation and identification of fungi :}

This study showed the isolation of several species of fungi associated with fodder grains, many of them were diagnosed : Aspergillus niger, Aspergillus flavus, Aspergillus ochraceus, Aspergillus fumigatus, Penicillium natatum, Rhizopus stolanifer, Alternaria alternata, Fusarium solani, Fusarium proliferatum, Fusarium sp. Tricoderma sp. . The results showed in Table No. ( 3 ) that there are some differences in the frequency of fungi that have been diagnosed in fodder grains that are non-sterilized and superficially sterilized. The fact that sodium hypochlorite is sterile and its effect is limited to surface fungi carried on fungi-contaminated grains. These results are consistent with what [19] . That confirmed the effect of sodium hypochlorite on externally carried fungi . The results also indicate that the Aspergillus niger percentage is the highest frequency in the treatment surficial of non-sterilized grains of this fungus about $15.23 \%$, frequency in the sterilized treatment was $13.88 \%$ for same fungus , the reason is due to the ability of this fungus to form large numbers of reproductive units even in inappropriate environmental conditions, as well as its small diameters less than 15 nanometers and thus a great ability to spread and contaminate grains. As for A.flavus fungus, its frequency in treating superficially non-sterilized and sterilized grains was $13.01 \%$ and $15.87 \%$ respectively. These results are consistent with what he mentioned [20], who confirm that Aspergillus species often top the fungi isolates from seeds. The frequency percentage of fungi $P$. notatum was $10.24 \%$ in the treatment of non-sterilized grains and $9.92 \%$ in the sterilized grain. These results are consistent with [21] mentioned that the fungi accompanying grains are $P$. notatum, A. alternate, etc. The A. alternata fungi are among the most important contaminated fungi for local and imported food products [22] which has a frequency of $8.58 \%$ in non- sterilized and $11.11 \%$ in the treatment of sterile grains. The contaminated fungus found in agricultural soils Rhizopus stolonifer its frequency percentage reached $9.69 \%$ and $1.98 \%$, respectively, in non-sterilized and sterile treatments . Fusarium solani, Fusarium proliferatum and Fusarium sp. The frequency in treatment of non-sterilized grains was $6.64 \%, 5.54 \%$ and $4.70 \%$, respectively, the percentage of these fungi in sterile treated $10.71 \%, 5.95 \%$ and $5.15 \%$ 
Table ( 3 ) : Fungi isolated from different fodder grains and their frequency percentage :

\begin{tabular}{|l|c|c|c|c|}
\hline \multirow{2}{*}{$\begin{array}{l}\text { Name of the isolated } \\
\text { fungus }\end{array}$} & \multicolumn{3}{|c|}{ Fungi percentages in different fodder grains } \\
\cline { 2 - 5 } & $\begin{array}{c}\text { Superficial non- } \\
\text { sterili }\end{array}$ & $\begin{array}{c}\text { Superficial } \\
\text { sterili }\end{array}$ & $\mathbf{X}^{\mathbf{2}}$ & P value \\
\hline Aspergillus niger & 15.23 & 13.88 & 1.074 & 0.3 \\
\hline A. flavus & 13.01 & 15.87 & 4.962 & $0.026^{*}$ \\
\hline A. ochraceus & 11.91 & 13.9 & 0.958 & 0.328 \\
\hline A. fumigatus & 11.8 & 10.71 & 0.102 & 0.749 \\
\hline Penicillium natatum & 10.24 & 9.92 & 0.088 & 0.767 \\
\hline Rhizopus stolanifer & 9.69 & 1.98 & 72.33 & $0 *$ \\
\hline Alternaria alternata & 8.58 & 11.11 & 5.434 & $0.020^{*}$ \\
\hline Fusarium solani & 6.64 & 10.71 & 16.084 & $0 *$ \\
\hline F. proliferatum & 5.54 & 5.95 & 0.234 & 0.628 \\
\hline Fusarium sp. & 4.70 & 5.15 & 0.322 & 0.570 \\
\hline Trichoderma sp. & 3.32 & 1.58 & 8.805 & $0.003^{*}$ \\
\hline X2 & 301.6 & 346.4 & & \\
\hline P value & $0 *$ & $0 *$ & & \\
\hline
\end{tabular}

$\mathrm{X} 2$ : Represents the value of the Chi square.

* significant differences at probability level $(\mathbf{P}<0.05)$.

\section{Molecular Diagnosis of Aspergillus niger :}

Measured purity and concentration DNA in A.niger strains :

The Table below shows the results of a DNA concentration and purity measurement for 4 strains .

\section{Table ( 4 ) : DNA concentration and purity for A. niger strains .}

\begin{tabular}{|c|c|c|}
\hline Strains & Concentration (ng / ML) & Purity \\
\hline $\mathbf{1}$ & 25.1 & 1.71 \\
\hline $\mathbf{2}$ & 23.8 & 1.68 \\
\hline $\mathbf{3}$ & 21.5 & 1.57 \\
\hline $\mathbf{4}$ & 18.3 & 1.75 \\
\hline
\end{tabular}

\section{Molecular Diagnostics using PCR :}

The fungus A.niger was selected to complete laboratory experiments on it and to support the diagnosis of the fungus use molecular diagnostics by PCR technology and to further confirm the validity of the diagnosis of fungus, use the primer 18S rRNA gene ITSI region for the A.niger fungus that was designed for study by the Primers 3 plus program and after obtaining on the genetic sequence of the primer of the gene bank GeneBank located on the website WWW.ncbi.nlm.nih.gov. The electrophoresis distance of A.niger doubled DNA with regions ITS-1 reach to (1500 bp) Which proved successful in the process of amplification with A.niger strains when the apperance of base pairs, and these are similar to the results of a study conducted by [23] when amplifying the DNA region ITS and its approval in the diagnosis of fungi as global genetic code . 


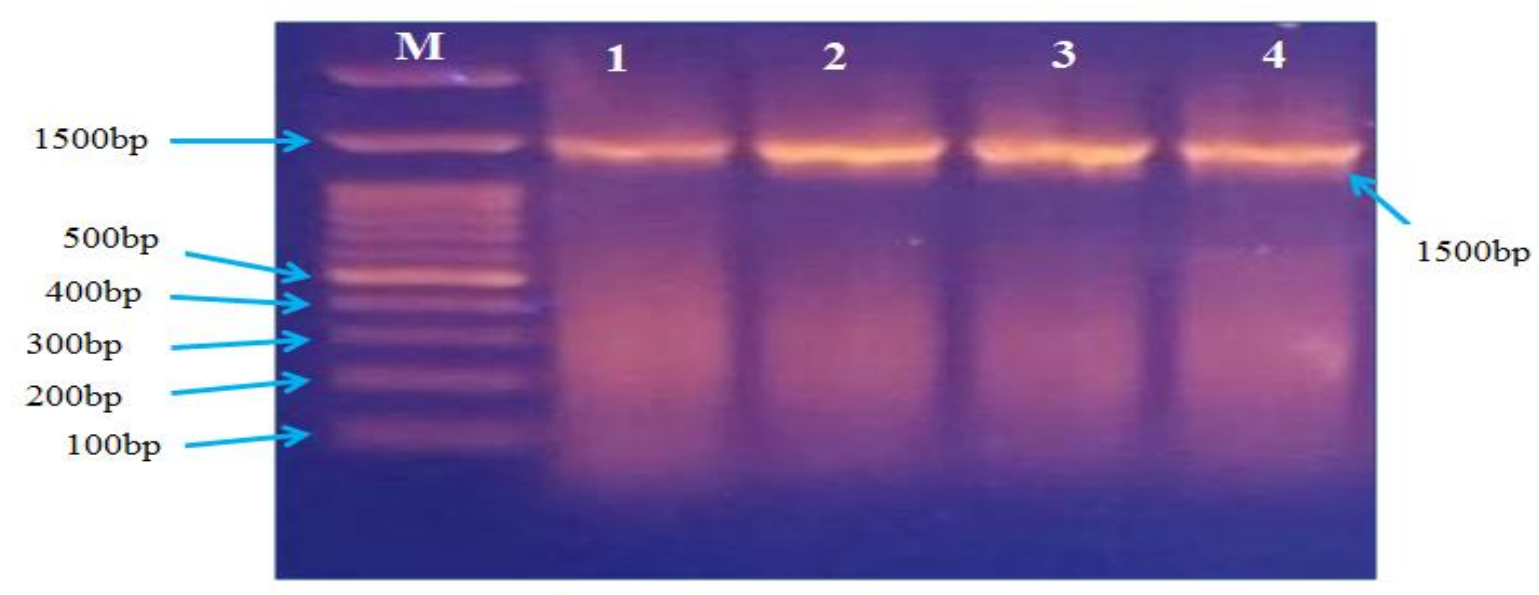

Figure ( 1 ) : The result of the electrophoresis using the Agarose gel (\% 1), at 70v for one hour, showing the results of the PCR examination of the 18S rRNA gene in the diagnosis of Aspergillus niger, where $M$ represents Ladder and (1- 4) represents A.niger strains .

At the molecular level of rRNA, an ITS region, which extends from ITS-1 and 5.8s to ITS-2, was selected to identify and diagnose some fungi, particularly some species of [24]. Phylogenetic tree analysis was performed using ITS-1 region sequences of local A.niger strains and compared with the same region sequences of some global and local A.niger strains registered in the NCBI after obtaining the nucleotide sequence of the DNA bundle of the local strain. Figure ( 2 ), ( 3 ).

1)TAGGATAGTGGCCTACCATGGTGGCAACGGGTAACGGGGAATTAGGGTTCGATTCCGGAGAGGGAGCCTG AGAAACGGCTACCACATCCAAGGAAGGCAGCAGGCGCGCAAATTACCCAATCCCGACACGGGGAGGTAGT GACAATAAATACTGATACGGGGCTCTTTTGGGTCTCGTAATTGGAATGAGTACAATCTAAATCCCTTAACGA GGAACAATTGGAGGGCAAGTCTGGTGCCAGCAGCCGCGGTAATTCCAGCTCCAATAGCGTATATTAAAGTT GTTGCAGTTAAAAAGCTCGTAGTTGAACCTTGGGTCTGGCTGGCCGGTCCGCCTCACCGCGAGTACTGGTCC GGCTGGACCTTTCCTTCTGGGGAATCTCATGGCCTTCACTGGCTGTGGGGGGAACCAGGACTTTTACTGTGA AAAAATTAGAGTGTTCAAAGCAGGCCTTTGCTCGAATACATTAGCATGGAATAATAGAATAGGACGTGCGG TTCTATTTTGTTGGTTTCTAGGACCGCCGTAATGATTAATAGGGATAGTCGGGGGCGTCAGTATTCAGCTGTC AGAGGTGAAATTCTTGGATTTGGCGAAGACTAACTACTGCGAAAGCATTCGCCAAGGATGTTTTCATTAATC AGGGAACGAAAGTTAGGGGATCGAAGACGATCAGATACCGTCGTAGTCTTAACCATAAACTATGCCGACTA GGGATCGGACGGTGTTTCTATTATGACCCGTTCGGCACCTTACGAGAAATCAAAGTTTTTGGGTTCTGGGGG GAGTATGGTCGCAAGGCTGAAACTTAAAGAAATTGACGGAAGGGCACCACCAGGCGTGGAGCCTGCGGCTT AATTTGACTCAACACGGGGAAACTCACCAGGTCCAGACAAAATAAGGATTGACAGATTGAGAGCTCTTTCT TGATCTTTTGGATGGTGGTGCATGGCCGTTCTTAGTTGGTGGAGTGATTTGTCTGCTTAATTGCGATAACGAA CGAGACCTCGGCCCTTAAATAGCCCGGTCCGCATTTGCGGGCCGCTGGCTTCTTAGGGGGACTATCGGCTCA AGCCGATGGAAGTGCGCGGCAATAACAGGTCTGTGATGCCCTTAGATGTTCTGGGCCGCACGCGCGCTACA CTGAC

2)CTAGTATAGCACTTTATACTGTGAAACTGCGAATGGGCTCATTAAATCAGTTATCGTTTATTTGATTAGAT AAAAAACCAATGCCCTTCGGGGCTCCTTGGTGAATCATAATAACTTAACGAATCGCATGGCCTTGCGCCGGC GATGGTTCATTCAAATTTCTGCCCTATCAACTTTCGATGGTAGGATAGTGGCCTACCATGGTGGCAACGGGT AACGGGGAATTAGGGTTCGATTCCGGAGAGGGAGCCTGAGAAACGGCTACCACATCCAAGGAAGGCAGCA GGCGCGCAAATTACCCAATCCCGACACGGGGAGGTAGTGACAATAAATACTGATACGGGGCTTTTGGGTCT CGTAATTGGAATGAGTACAATCTAAATCCCTTAACGAGGAACAATTGGAGGGCAAGTCTGGTGCCAGCAGC CGCGGTAATTCCAGCTCCAATAGCGTATATTAAAGTTGTTGCAGTTAAAAAGCTCGTAGTTGAACCTTGGGT CTGGCTGGCCGGTCCGCCTCACCGCGAGTACTGGTCCGGCTGGACCTTTCCTTCTGGGGAATCTCATGGCCTT CACTGGCTGTGGGGGGAACCAGGACTTACTGTGAAAAAATTAGAGTGTTCAAAGCAGGCCTTTGCTCGAAT ACATTAGCATGGAATAATAGAATAGGACGTGCGGTTCTATTTTTGTTGGTTTCTAGGACCGCCGTAATGATT AATAGGGATAGTCGGGGGGCGTCAGTATTCAGCTGTCAGAGGTGAAATTCTTGGATTTGCTGAAGACTAACT ACTGCGAAAGCATTCGCCAAGGATGTTTTCATTAATCAGGGAACGAAAGTTAGGGGATCGAAGACGATCAG ATACCGTCGTAGTCTTAACCATAAACTATGCCGACTAGGGATCGGACGGTGTTTCTATTATGACCCGTTCGG CACCTTACGAGAAATCAAAGTTTTTGGGTTCTGGGGGGAGTATGGTCGCAAGGCTGAAACTTAAAGAAATT 
GACGGAAGGGCACCACCAGGCGTGGAGCCTGCGGCTTAATTTGACTCAACACGGGGAAACTCACCAGGTCC AGACAAAATAAGGATTGACAGATTGAGAGCTCTTTCTTGATCTTTTGGATGGTGGTGCATGGCCGTTCTTAG TTGGTGGAGTGATTTGTCTGCTTAATTGCGATAACGAACGAGACCTCGGCCCTTA

Figure ( 2 ) : The nucleotide sequence of the DNA (PCR - amplified product) multiplication from the A.niger fungus . The strains of A.niger fungus were registered at the NCBI National Center under the first Accession number (MT644083) and the second (MT644084) at the GenBank .

After conducting the molecular diagnosis of A. niger by using 18s RNA gene primer, the PCR product was sent to South Korea to conduct a nucleotide sequencing analysis for strains of this fungus and shown in Figure (2), and after the phylogenetic tree was drawn using the Mega 10 program, it was noticed that the strains of this fungus spread worldwide, the genetic similarity ratio between the strains of this fungus with the other of the global strains ranged between (97-100\%) that registered in (NCBI) in the following countries (MH532513 India, GQ338836 Iran, AF548064 Sweden, DQ915806 China, MG211803 Nigeria, MN420840 Pakistan, KT832783 USA, MF072566 Portugal) , the phylogenetic tree represents the relationship between a gene or protein sequence, or between the same species or genus .

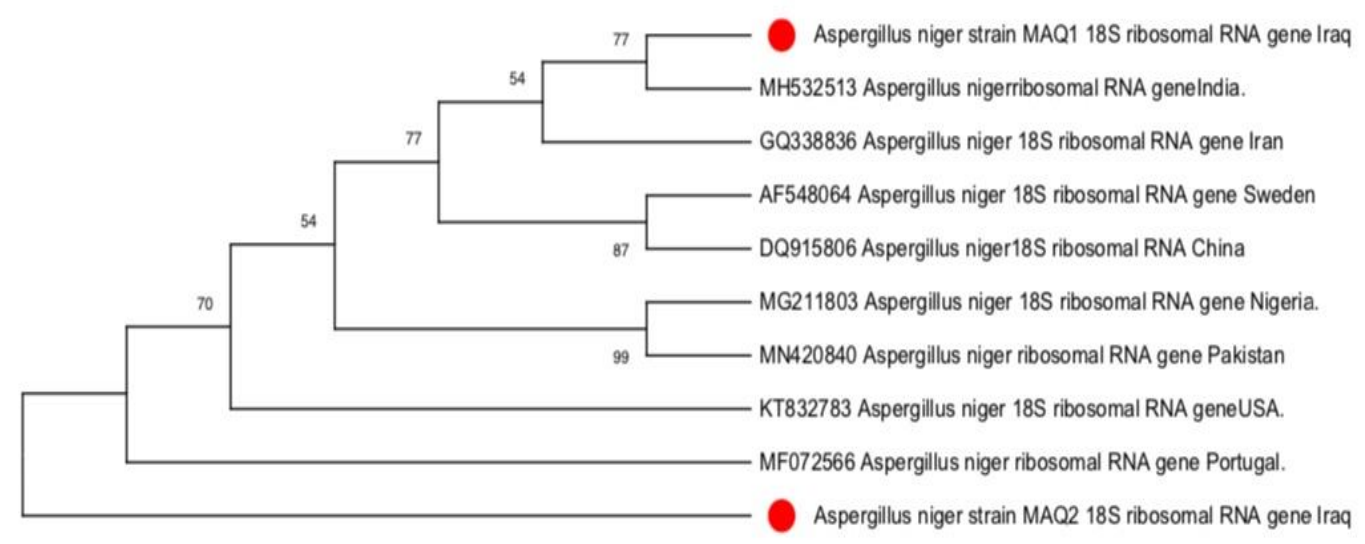

Figure ( 3 ) : Shows the Phylogenetic tree analysis of $A$. niger fungus using the Mega 10 program , and that the red mark indicates of two strains of $A$. niger fungus isolated in Iraq •

\section{Effect of P.ostreatus on growth of A.niger contamination on PDA : (Antagonism)}

The results of the study of the anti-fungal ability of P.ostreatus against fungus isolated from fodder grains showed the ability of the P.ostreatus fungus to completely inhibit the growth of $A$. niger fungus , as was observed on the culture medium PDA, and the degree of antagonism was calculated according to the five-scale standard mentioned by [15] .These results are consistent with what [25] showed, which observed the nature of the antagonism between P.ostreatus and other contaminated or pathogenic fungi. Perhaps the reason is due to the high ability to antagonism P.ostreatus against other pathogenic fungi through its direct parasitism on fungal Mycelium in addition to its ability to analyze cell walls by secreting different analyzing enzymes or producing antibiotics, and one of the other reasons that explain this high antagonism ability is competition for food and place in addition to the speed of its growth in the medium when the longer the incubation period. 


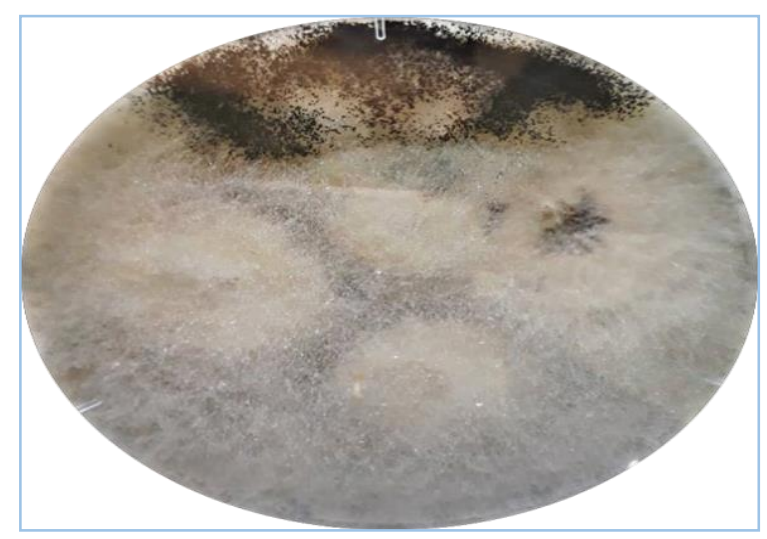

Figure ( 4 ) : Show ability of P.ostreatus to inhibit or reduce growth of A.niger fungus when antagonism occurs between two fungus on solid medium (PDA) after the incubation period ends , Where the white color in the petri indicates P.ostreatus, while the black growing it is A.niger .

\section{Effect of $P$. ostreaus filtrate concentrations on the radial growth of $A$. niger on PDA :}

The results in Table (5) showed significant differences in the treatment of $P$. ostreaus filtrate, as the highest inhibition rate of the contaminated A. niger in the medium containing a concentration of $30 \%$ of the $P$. ostreaus filtrate, where the average diameters of colonies was $4.83 \pm 0.25 \mathrm{~cm}$, and inhibition percent reached $44.43 \%$ in the growth of tested fungus at a concentration of $10 \%$, and the average diameters of colonies $3.93 \pm 0.25 \mathrm{~cm}$ with inhibition percent $54.78 \%$ at a concentration of $20 \%$, while the growth rate of colonies diameters reached $3.21 \pm 0.03 \mathrm{~cm}$ and inhibition percent reach to $63.06 \%$ in concentration $30 \%$, compared to the average diameters of colonies in the control treatment , $8.7 \pm 0 \mathrm{~cm}$ . It is very clear the effect of the concentrations of different $P$. ostreaus filtrate on the contaminated fungus for feeds .

The effect of calcium citrate on the radial growth of $A$. niger fungus on PDA :

The results showed in Table (5) that significant differences occurred for the treatment of calcium citrate, as the highest rate of inhibition of the contaminated A. niger fungus appeared in the medium containing the concentration of $30 \%$ of calcium citrate, as the average colonies diameters was $4.99 \pm 0.12 \mathrm{~cm}$ and reached inhibition percent $42.63 \%$, while the lowest rate of inhibition in concentration $10 \%$, as the average diameters of colonies was $5.8 \pm 0.06 \mathrm{~cm}$, with inhibition percent about $33.25 \%$, while in concentration $20 \%$, the growth rate of colonies diameters reached $5.52 \pm 0.05 \mathrm{~cm}$, with inhibition percent $36.51 \%$ compared to the average diameters of the colonies in the control treatment was $8.7 \mathrm{~cm}$. The reason may be that the calcium compounds, including the calcium citrate influence value of the $\mathrm{pH}$ toward the basal in PDA medium, which negatively affects the growth of fungi in different media , these results are consistent with [22] when he conducted a study on the possibility of using one of the compounds of calcium to control fungi contaminated with foods .

The interfere effect of calcium citrate and $\boldsymbol{P}$. ostreaus filtrate on the radial growth of $A$.niger fungus on PDA :

The interference results showed of calcium citrate with $P$. ostreaus filtrate on the radial growth of the isolated $A$. niger fungus in Table (5) indicated that significant differences occurred between the different concentrations of $P$. ostreaus filtrate and calcium citrate and significantly affected the growth of the contaminated $A$. niger fungus so that the rates of diameters colonies are inversely proportional with concentration of both the filtrate and citrate. Observed a decrease in the colonial diameters of the contaminated fungus as interfere concentration increases. The superiority was observed in the results of the fungus filtrate and the chemical material inhibiting the radial growth of the contaminated fungus when comparing and using each them separately . as the average diameters of the contaminated fungus colonies at a concentration of $30 \%$ for this treatment was $2.45 \pm 0.005 \mathrm{~cm}$, with inhibition 
percent about $71.79 \%$ which is more effective than each other treatment, but at concentrations of $20 \%$ , the average diameters of the colonies reached $2.53 \pm 0.02 \mathrm{~cm}$ with inhibition percent $70.87 \%$ while the average diameters of the colonies at a concentration of $10 \%$ was $3.21 \pm 0.05 \mathrm{~cm}$ and the inhibition percent was $63.06 \%$ compared to the average diameters of the colonies in the control treatment $8.7 \pm$ $0 \mathrm{~cm}$.

Table ( 5 ) The effect of calcium citrate and $P$. ostreaus filtrate on the radial growth of $A$. niger .

\begin{tabular}{|c|c|c|c|c|c|c|c|}
\hline \multirow{2}{*}{ Con. } & \multicolumn{2}{|c|}{$\begin{array}{c}\text { Calicum citrate } \\
\text { treatment }(1)\end{array}$} & \multicolumn{2}{|c|}{$\begin{array}{l}\text { P.ostreatus Filtrate } \\
\text { treatment( } 2)\end{array}$} & \multicolumn{2}{|c|}{ Interfere between $1+2$} & \multirow{2}{*}{$\begin{array}{c}\text { C. Inhibition average } \\
\pm \text { S.D }\end{array}$} \\
\hline & $\begin{array}{l}\text { Diameter } \\
(\mathrm{cm})\end{array}$ & Inhibition \% & Di. $(\mathrm{cm})$ & In. $\%$ & Di. (cm) & In. $\%$ & \\
\hline $10 \%$ & $5.8 \pm 0.06$ & $33.25 \pm 0.75$ & $4.83 \pm 0.25$ & $44.43 \pm 2.89$ & $3.21 \pm 0.05$ & $63.06 \pm 0.63$ & $46.91 \pm 15.05$ \\
\hline $20 \%$ & $5.52 \pm 0.05$ & $36.51 \pm 0.58$ & $3.93 \pm 0.25$ & $54.78 \pm 2.89$ & $2.53 \pm 0.02$ & $70.87 \pm 0.28$ & $54.05 \pm 17.19$ \\
\hline $30 \%$ & $4.99 \pm 0.12$ & $42.63 \pm 1.44$ & $3.21 \pm 0.03$ & $63.06 \pm 0.40$ & $2.45 \pm 0.005$ & $71.79 \pm 0.06$ & $59.15 \pm 14.96$ \\
\hline Control & $8.7 \pm 0$ & & $8.7 \pm 0$ & & $8.7 \pm 0$ & & \\
\hline \multicolumn{2}{|c|}{$\begin{array}{c}\text { Inhibition average of } \\
\text { treatment } \pm \text { S.D }\end{array}$} & $37.46 \pm 4.76$ & & $54.08 \pm 9.31$ & & $68.57 \pm 4.79$ & $53.37 \pm 14.65$ \\
\hline L.S.D & Con. & 1.491 & Treat. & 1.491 & Inter. & 2.582 & \\
\hline
\end{tabular}

* significant differences at probability level $(\mathbf{P}<0.05)$.

\section{Conclusion}

The current study reached Aspergillus niger fungus are issued first for fungi isolated from fodder grains, which were sterilized superficially, and third for fungi isolated from superficially sterilized grains, molecular diagnosis using PCR with sequencing analysis and phylogenetic tree mapping for the purpose of comparing A.niger strains with some global strains . The results of two treated showed a clear decrease in the level of colonial diameters, where the rate of inhibition reached $71.79 \pm 0.06 \%$ at concentration $30 \%$, the effect of the p.ostreatus was better than the calcium citrate when treating each one separately .

\section{Recommendation}

Repeated examination of the stored feed for birds and poultry and ensure that optimal storage conditions are observed and thus reduce or limit the spread of fungi producing various toxins because they have dangerous effects on human health also use of P.ostreatus with food and feed because of its great role in overcoming toxic contaminated fungi , use of calcium citrate in the treatment of grains included in the feeds or as feed additives. With the investigation of Pleurotus ssp. that may have the ability to inhibit contaminated toxic fungi .

\section{References}

[1] Makun , H. A., S. T. Anjorin , B.Moronfoye, F. O. Adeje, O. A. Afolablil, G. Fagbayibo , B.O. Balogum and Surajudeen A.A.(2010). Fungal and aflatoxin contamination of me human food commodities in Nigeria. Afiican Journal of Food Science , 4:127-135.

[2] Sulaiman , E.D.(1977) - Comprehensives survey of fungi associated with in Iraq note on pathogenicity and control. M.Sc Thesis, College of Agriculture and Foresty, Mosul, Iraq .

[3] Abbas , H. K.; C. J. Mirocha, R. A.; Meronuck, J. D.; Pokorny, S. L.; Gould, and Kommedahl, T. (1988). Mycotoxins and Fusarium spp Associated with infected Ears of corn in Minnesota. Appl . Enviromental Microbiology. 54 (8): 1930 - 1933.

[4] Salvaggio , J . I. (2006) .Extrinsic allergic alveolitis (hypersensitivity - pneumonitis): Past , Present and future . Clin . EXP. Allergy $27: 18-25$.

[5] Pitt, J.I. and Hocking ,A.D.(1985) . Fungi and Food Spoilage. Academic Press, Sydney, Australia. 
[6] Korzeniowska - Kosela,M.; Domej,J.; Herman, R. ; Krause , M . and Maier,A. (1990). Pulmonary Aspergillosis caused by Aspergillus. Pneumonal . Pol. 58 (1) : $328-333$.

[7] Abdul-hamid, Abdul-hamid Mohamed. (2000). Fungi and Mycotoxins. University Publishing House - Egypt .

[8] Collee , J. G.; Fraser , A.G. ;Marmion, B.P. and Simmons,A.(1996). Parctical Medical Microbiology .14 ted . Makie and Macarthy, Pearson Professional Limited .

[9] Saadon, A.A. S. (2005). Use of Medicago sativa root powder and Sodium Hypochlorite as alternatives on the use of chemical pesticides to control fungi associated with wheat seeds before agriculture . Al-Qadisiyah Journal of Pure Sciences. Environmental Research Issue. Vol. 144-136 (10).

[10] Mikhail, S. and Beder, T. (1982). Seed diseases. Ministry of Higher Education and Scientific Research. University Mosul.

[11] Quinn, P.J.; Carter . M.G.; markey , B.and Carter , G.R.(2002). Clinical Veterinary Microbiology, M. Wlof, London .

[12] De Hoog , G. S.; Guarro, J.; Gené, J.; and Figueras, M. J. (2000). Atlas of clinical fungi, 2nd. Utrecht, The Netherlands: Centra albureau voor Schimmel cultures, 305-310 .

[13] Carvallo , A. ; Furlanetto, L.V. S.; Maruyama, F.H. ; Nakazato, L. and Paschoalin V.M (2015) . Evaluation of The efficiency of Nested Q- Pcr In the detection Of Mycobacterium Tuberculosis complex directly from Tuberculosis-suspected Lesions In post-Mortem Macroscopic inspections of Bovine carcasses slaughtered In The State Of Mato Grosso, Brazil . US National Library of Medicine National Institutes of Health .

[14] Sambrook , J. and Green M. (2012) . Molecular cloning A laboratory Manual . 4th ed . cold spring Harbor Laboratory Press, N . Y.

[15] Bell , D. K. ;Well , H.O. and Markham , C . R. (1982). In vitro antagonisms of Trichoderma sp. Against six fungeal plant pathogens. Phytopathololgy , 72 pp : $379-382$.

[16] Dixit , S. N. ; Tripathy , S. C. and Upadyyey ,R.R. (1976) . The antifungal substasnce of rose flower (Rose indica) Economic Botany. 30:371-373 .

[17] Shaaban, A. and Al-Mallah, N. M. (1993). Pesticides. House Al-Ketub Printing and Publishing Est. University of Al Mosul .

[18] Al-Musawi, A. A.H. (2013). Efficiency of some biological and chemical treatments in controlling the growth of Fusarium oxysporium, f.sp.lycopersici associated with tomato seeds and roots. Master Thesis . College of Science - Al-Qadisiyah University.

[19] Sarhan, A. R. T.; Mohsen, K. Y.and Saadoun, A.A.S. (2001). a study Efficiency of wheat and barley seeds in several regions in the governorates of Al-Qadisiyah and Wasit. Al-Qadisiyah Magazine, Vol 6, Issue 94-83.

[20] Al-Baldawi , M. S. ;Mohsen, H.;Halima, Z. and Al-Adil, Kh. M. (2009). The efficacy of urea, flux and powders of some medicinal plants in inhibiting the growth of two species of fungus Aspergillus in cultural media. Iraqi Journal of Agricultural Sciences .

[21] Jaber, K. S. (2004). Detection of fungi transferred by Sesamum indicum seeds in Iraq Pathological importance and resistance. Iraqi Agricultural Science Journal, Volume 35, Number 3 p: 110-103.

[22] Al-Saeedi, Gh. H. (2018). Evaluation of the role of fungus Pleurotus ostreatus and CaCO3 in the control of Alternaria alternata fungi isolated from some local and imported food products in the city of Diwaniyah. Master Thesis . College of Science - Al-Qadisiyah University.

[23] Muhammad, M. N. and Alaa, H. I. (2016). Genotyping of some fungal strains an region ITS interal transcribed spacer as global genetic code .

[24] Schoch, C.L.; Seifert, K.A.; Huhndorf, S.; Robert, V.; Spouge, J.L.; Levesque, C.A. Chen, W. (2012). Nuclear ribosomal internal transcribed spacer (ITS) region as a universal DNA barcode marker for fungi. Proc Natl Acad Sci U S A 109:6241-6246. 
[25] Al-Jubouri, I. W. A. (2011). Improve the yield and safe capacity of Pleurotus ostreatus using Mineral and organic nutrients . Iraqi Agricultural Science Journal 72-65: (6) 42. 